\title{
P148 TRIAGE
}

Jane Wythe St Nicholas Hospice Care, Bury St Edmunds, UK

10.1136/bmjspcare-2011-000105.148

A hospice first response

Introduction The project focused on introducing a clinical nurse specialist as the triage lead for a hospice service offering day care, inpatient and community care. Referrals had previously been managed by an administrator with clinical support.

\section{Aims}

- To ensure timely responses to patient and family needs and ensure the organisation's response to referrals was appropriate

- Provide meaningful support to professional and lay referrers with a consistent response.

Methods The triage specialist led a rigorous evaluation of the referral process while supportively challenging local referral culture; funding for the triage post was secured from a Big Lottery Fund grant.

- Clinical Nurse Specialist and administrative support developed robust systems and processes

- Education strategies were employed with local referrers using team and one to one approaches

- Professional and lay carer opinion was actively sought through the use of questionnaires.

\section{Results}

- The triage approach became a catalyst for a new organisational strategy 2011, because of the identified unmet need

- Local regional professionals reported a consistency and transparency in clinical decision-making by the hospice

- Hospice staff expressed an increased confidence in first assessment allocation which has indirectly upheld the distinct contributions of each discipline

- Average response time to referral reduced to from 1 to 2 days to under 1 day.

Discussion and conclusion An experienced practitioner as triage lead has enabled further exploration of allocation of first assessment to skill mix within disciplines including healthcare assistants. Examination of triage identified that it was more of a first response with a telephone assessment.

The processes established has quickly become embedded in the Hospice's practice culture and remains a source of pride and comfort during periods of rising public and professional expectation of hospice services. 